\title{
Analysis free surface of nonlinear seepage using the MQRBF method
}

\author{
Yan $\mathrm{SU}^{1, \mathrm{a}^{*}}$,Zhi-ming ZHENG ${ }^{1, \mathrm{~b}}$, Cheng-yu $\mathrm{GU}^{2}$, Long-teng ZHANG ${ }^{3}$ \\ ${ }^{1}$ College of Civil Engineering, Fuzhou University,350116, Fuzhou, China \\ ${ }^{2}$ Department of Harbor and River Engineering, Taiwan Ocean University, 20224, Keelung, China \\ ${ }^{3}$ POWERCHINA HUADONG ENGINEERING CORPORATION LIMITED,310014, Hangzhou, China
}

\begin{abstract}
In order to solve the characteristics of low accuracy and slow efficiency in traditional numerical solution the free surface problem, the multiquardatic radial base function collocation method(MQ RBF) is used to analyze the constant seepage and unsteady seepage of the homogeneous earth dam. Computation of transient problem of free surface of earth dam by the linear derivation of Richards equation. The results show that the calculation accuracy of the MQRBF is higher than that of the traditional numerical method. The solution process does not involve numerical integral calculation and grid reorganization, which greatly reduces the calculation amount. Compared with the Trefftz method, it has the advantage of solving boundary values and internal values at the same time. It is not limited by the solution of the Laplace equation, and its application is wider and simpler.
\end{abstract}

\section{Preface}

Solving the free surface of seepage is the key and difficult point of geotechnical engineering research. This nonlinear problem of seepage area ${ }^{[1]}$ is a prerequisite for studying the stability of dams and mountain slopes. In the conventional analysis, the main solving methods include boundary element method, finite element method, finite difference method and meshless method. In order to improve and overcome the time consumed by traditional numerical methods in calculations, grid limitations and error accuracy. In recent years, the meshless method has been proposed and applied by many scholars. It is characterized by no need to generate grids in numerical calculations, coordinates can be arbitrarily distributed, various complex research areas can be calculated and simulated, and it has the advantages of high accuracy and good convergence.

Common meshless methods include basic solution method, Trefftz method and radial basis function collocation method; this paper uses multivariate quadratic radial basis function collocation method which can directly meet the governing equations and boundary conditions at the discrete points to solve the nonlinear problem of the seepage area of the reservoir water level change in the glycerin test model ${ }^{[3]}$, and compare multiple methods ${ }^{[4]}$ to verify the feasibility and accuracy of the multivariate quadratic radial basis function collocation method (MQ RBF) seepage analysis model to solve the nonlinear seepage free surface problem.

\section{Meshless Method}

\subsection{Mathematical model}

The basic equation of percolation is based on Darcy's law, derive the Richard equation of two-dimensional unsteady seepage.

$$
\nabla K \nabla H(x, z)=K_{x} \frac{\partial^{2} H}{\partial x^{2}}+K_{z} \frac{\partial^{2} H}{\partial z^{2}}=\frac{\partial H}{\partial t}
$$

Where $H$ is total head; $K$ are constants with the same permeability coefficient in the $\mathrm{x}$ and $\mathrm{z}$ directions for all homogeneous seepage problems. The boundary conditions of the formula are usually divided into 3 categories:

Constant head boundary

$$
\left.H\right|_{\Gamma_{3}, \Gamma_{5}}=h, o n \Gamma_{3} \& \Gamma_{5}\left(\Gamma_{4} \& \Gamma_{6}\right)
$$

Constant flow boundary

$$
\frac{\partial H(x, z)}{\partial n}=0, \text { on } \Gamma_{4}\left(\Gamma_{5}\right)
$$
flow

Mixed boundary of constant head and constant

$$
\left\{\begin{array}{c}
H(x, \mathrm{z})=h, o n \Gamma_{1} \& \Gamma_{2}\left(\Gamma_{1} \& \Gamma_{2} \& \Gamma_{3}\right) \\
k_{n}\left(\frac{\partial H(x, z)}{\partial n}\right)=0, \text { on } \Gamma_{1} \& \Gamma_{2}
\end{array}\right.
$$

Where $n$ is direction cosine of the normal outside the boundary along the coordinate direction, $k_{n}$ is permeability coefficient along the normal vector. (Unsteady seepage boundary in brackets) 


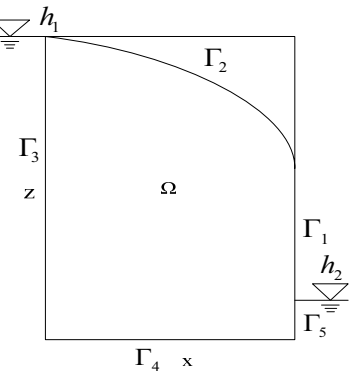

(a) Steady seepage

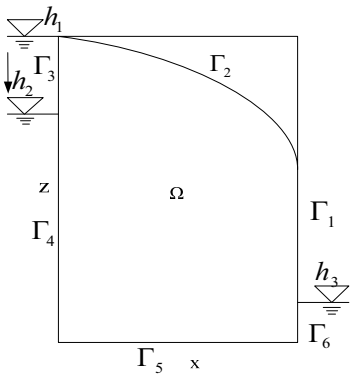

(b) Unsteady seepage
Fig.1 Earth dam glycerin experimental model diagram

In Figure 1, $\mathrm{x}=4 \mathrm{~m}, \mathrm{z}=6 \mathrm{~m}, \Gamma_{3 \mathrm{a}} \& \Gamma_{5 \mathrm{a}}$ and $\Gamma_{4 \mathrm{~b}} \& \Gamma_{6 \mathrm{~b}}$ are the upstream water head boundaries and the downstream water head boundary, which belong to the fixed-head boundary; $\Gamma_{4 \mathrm{a}}$ and $\Gamma_{5 \mathrm{~b}}$ are the boundaries of the horizontal dam foundation of the impervious earth dam, which belongs to the boundary condition of constant flow. In (a), since the free liquid surface is a streamline and its normal velocity is $0(\mathrm{~m} / \mathrm{s})$, the boundary $\Gamma_{1 \mathrm{a}} \& \Gamma_{2 \mathrm{a}}$ is not only a constant head condition, but also a constant flow boundary condition. In (b), as the upstream water level changes, the flow velocity also changes. Therefore, $\Gamma_{1 \mathrm{a}} \& \Gamma_{2 \mathrm{a}} \& \Gamma_{3 \mathrm{a}}$ boundaries belong to the Dirichlet boundary condition, $\Omega$ belongs to the control domain.

\subsection{Radial base function collocation method}

We must first give an initial guess $\left(x_{0}, z_{0}\right)$ and then iterative calculation. We use multiple quadratic Radial basis function collocation method for discrete solution:

$$
\begin{aligned}
& H(x, z)=\sum_{j=1}^{N} \lambda_{j} \phi\left(r_{i j}\right)= \\
& \sum_{j=1}^{N} \lambda_{j} \sqrt{\left(r_{i j} \varepsilon\right)^{2}+1}(i, j=1,2,3 \cdots N)
\end{aligned}
$$

Where $i, j$ is the node number of the collocation point; $\phi(r)$ is the radial basis function; $\lambda_{j}$ is the undetermined interpolation coefficient; $r_{i j}$ is the Euclidean distance from the node to the node.

Substitute the formula (5) obtained after the second differentiation in the $\mathrm{x}$ and $\mathrm{z}$ directions into the formula (1) discrete and the time discretization is solved by the display difference method.

$$
K_{x} \sum_{j=1}^{N} \lambda_{j} \frac{\varepsilon^{2}}{\left[\left(r_{j} \varepsilon\right)^{2}+1\right]^{3 / 2}}+K_{z} \sum_{j=1}^{N} \lambda_{j} \frac{\varepsilon^{2}}{\left[\left(r_{j} \varepsilon\right)^{2}+1\right]^{3 / 2}}=\frac{\partial H}{\partial t}
$$

\section{Analysis of examples}

\subsection{Steady seepage free surface problem}

\subsubsection{The solving process of the seepage free surface problem in the steady condition}

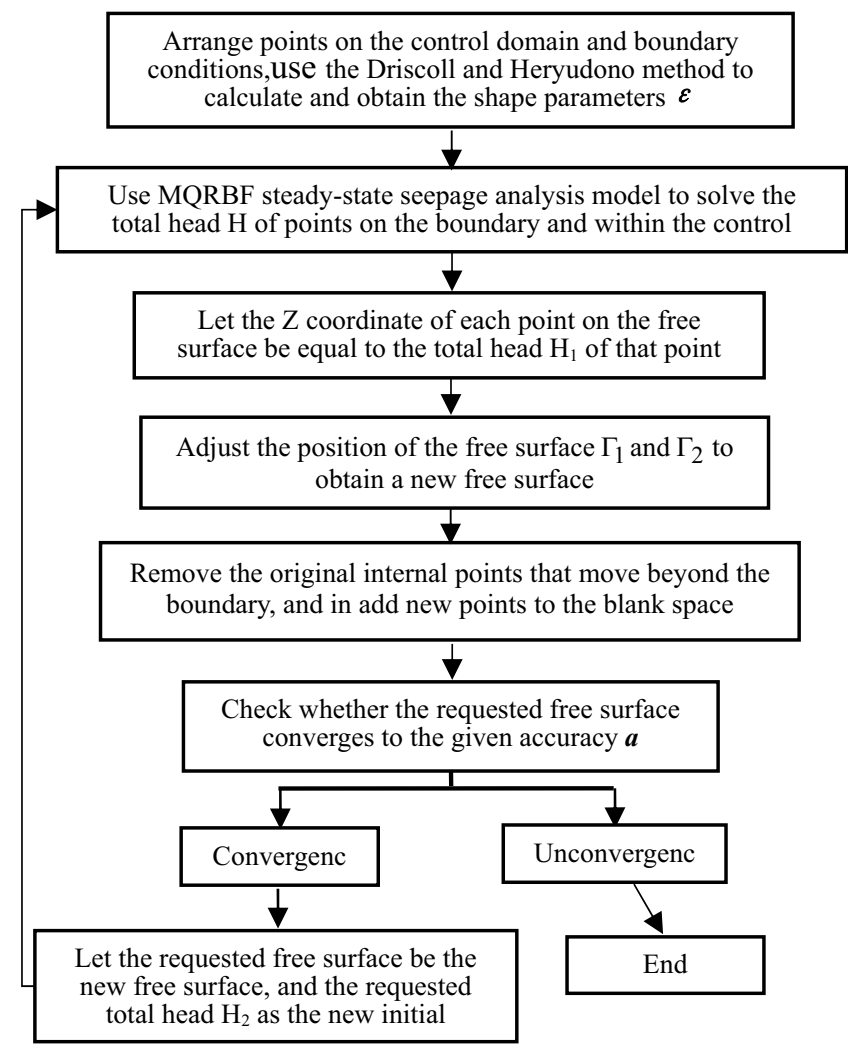

Fig.2 The computational flowchart of steady seepage with free surface

This case is based on the glycerin experiment in Mao Changxi's "Seepage Calculation Analysis and Control" book $^{[3]}$, considering the seepage of a rectangular homogeneous earth dam on an impervious foundation, the upstream water level is $6 \mathrm{~m}$, the downstream water is $1 \mathrm{~m}$, and the soil permeability coefficient of the earth dam is $K x=K z=1.39 \times 10^{-4} \mathrm{~cm} / \mathrm{s}$. According to the collocation method, 675 points are arranged inside the control domain, and 30, 90, 60, 30 and 15 points are arranged on the five boundaries of $\Gamma_{1}, \Gamma_{2}, \Gamma_{3}, \Gamma_{4}$ and $\Gamma_{5}$ respectively. Layout 900 points.

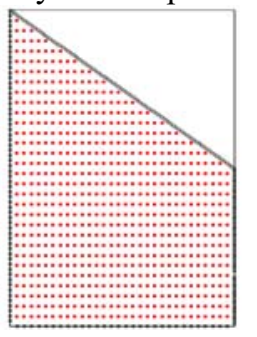

(a) original

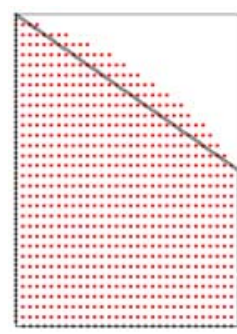

(b) remove

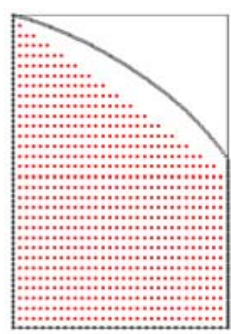

(c) add
Fig.3 The distribution map and point increase or decrease of seepage area

To set the accuracy $\alpha=10^{-4}$, the program obtains the 
convergence result after 29 iterations, and the required seepage free surface and the total head distribution of the seepage field are shown in Figure 4.

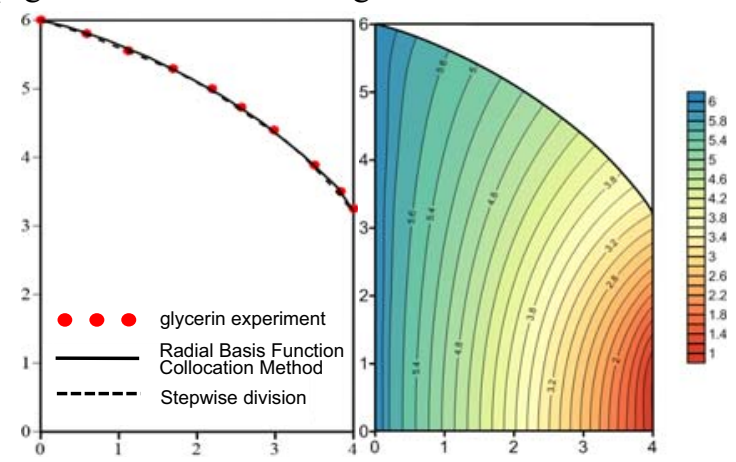

Fig.4 Free surface of seepage and total head distribution of seepage field

\subsubsection{Compare the results of this paper with experimental results and other numerical simulations}

Table 1 Comparison of typical positions of free surface of seepage flow

\begin{tabular}{cccccc}
\hline $\begin{array}{c}\text { Typical } \\
\text { position/m }\end{array}$ & $\mathrm{X}=0.5$ & $\mathrm{X}=1.5$ & $\mathrm{X}=2.5$ & $\mathrm{X}=3.5$ & $\mathrm{X}=4$ \\
\hline $\begin{array}{c}\text { glycerin } \\
\text { experiment/m }\end{array}$ & 5.82 & 5.37 & 4.74 & 3.82 & 3.25 \\
$\begin{array}{c}\text { Stepwise } \\
\text { division/m }\end{array}$ & 5.86 & 5.35 & 4.72 & 3.79 & 3.20 \\
$\begin{array}{c}\text { Calculated } \\
\text { value/m }\end{array}$ & 5.85 & 5.38 & 4.76 & 3.86 & 3.22 \\
Error/\% & 0.52 & 0.18 & 0.42 & 1.04 & 0.92 \\
\hline
\end{tabular}

Table 2 Comparison of calculation results of the height of overflow points by various calculation methods

\begin{tabular}{ccc}
\hline Method & $\begin{array}{c}\text { Overflow point } \\
\text { height/m }\end{array}$ & $\begin{array}{c}\text { Error with test } \\
\text { value/\% }\end{array}$ \\
\hline glycerin experiment & 3.25 & - \\
Finite element method & 3.31 & 1.85 \\
Trefftz direct method & 3.28 & 0.92 \\
Trefftz indirect method & 3.24 & 0.31 \\
Stepwise division method & 3.30 & 1.54 \\
Result & 3.22 & 0.92 \\
\hline
\end{tabular}

(1) From the perspective of solution accuracy, the meshless method has a certain improvement over the traditional numerical method of meshing. Trefftz direct method has the highest solution accuracy. While it can only solve Laplace equation, so its application is limited.

(2) From the perspective of solution efficiency, MQ RBFCM is based on the principle of interpolation, the solution process does not involve numerical integration calculation, the difficulty is lower, and the numerical simulation is easier to implement.

\subsection{Unsteady seepage free surface problem}

\subsubsection{The solving process of the seepage free surface problem in the unsteady condition}

For unsteady seepage flow, $\Gamma_{1}, \Gamma_{2}$ and $\Gamma_{3}$ are unfixed moving boundaries, and the boundary condition that the constant flow rate of seepage free surface is $0(\mathrm{~m} / \mathrm{s})$ is no longer applicable. According to Darcy's law $v=k i$, the normal moving speed of the free surface of seepage can be obtained as $v_{n}$ :

$$
v_{n}=k_{n}\left(\frac{\partial H(x, y)}{\partial n}\right) \text { on } \Gamma_{1} \& \Gamma_{2} \& \Gamma_{3}
$$

According to the geometric relationship, the free surface of seepage and the displacement along the direction of gravity can be expressed as:

$$
\Delta z=v_{n} n_{z} \Delta t
$$

At the moment $t_{1}=\Delta t$, the position of the seepage free surface can be obtained by the following formula:

$$
z^{1}=z^{0}+\Delta z=z^{0}+v_{n} n_{z} \Delta t
$$

675 points are arranged inside the control domain, and $30,90,15,30,30$ and 15 points are arranged on the five boundaries of $\Gamma_{1}, \Gamma_{2}, \Gamma_{3}, \Gamma_{4}, \Gamma_{5}$ and $\Gamma_{6}$ respectively. Adjust the position of the free surface $\Gamma_{1}, \Gamma_{2}$ and $\Gamma_{3}$, obtain a new free surface. Finally, the total head distribution value at each time is shown in Figure 5.

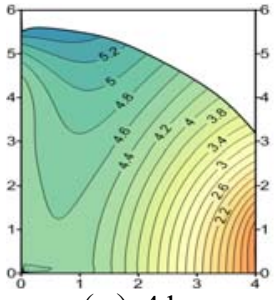

(a) $4 \mathrm{~h}$

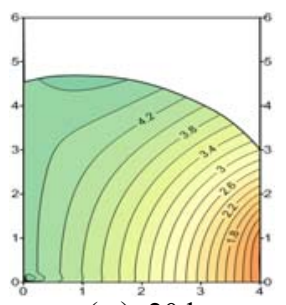

(c) $20 \mathrm{~h}$

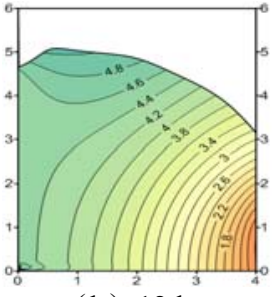

(b) $12 \mathrm{~h}$

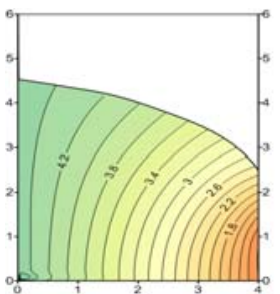

(d) $40 \mathrm{~h}$

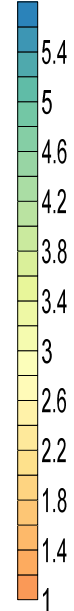

Fig.5 Distribution of total head at different time of unsteady seepage flow

\subsubsection{Compare of the results of this paper with other numerical simulation results}

By comparing the calculation results in Figure 6, after the water level drops sharply, the free surface calculated by MQ RBF is very similar to the Trefftz direct method, but have difference in calculation time. The solution result of the Trefftz indirect method is more consistent with the calculation result of MQ RBF. While after the water level drops sharply, both sides of the free surface drop at the same time, it is slightly unreasonable. The result of MQ RBF calculation of unsteady seepage free surface problem is reasonable . 


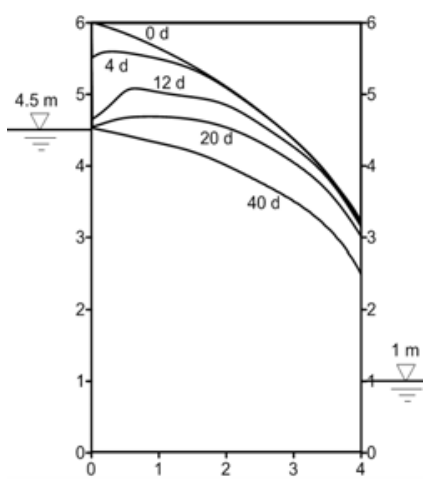

(a) MQ RBF

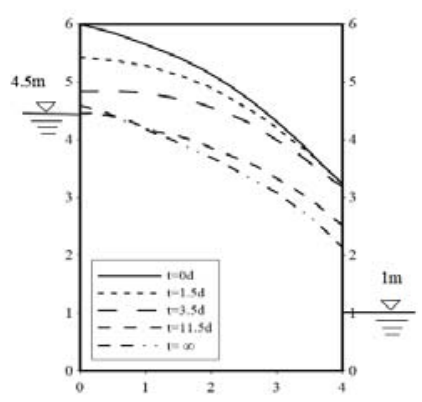

(b) Direct Trefftz

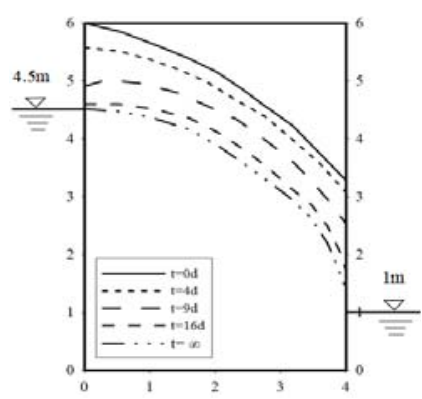

(c) Indirect Trefftz

Fig.6 The variation diagram of unsteady seepage with free surface

\section{Conclusions}

(1) When the multivariate quadratic radial basis function collocation method solves the nonlinear problem in the seepage area, its solution accuracy is significantly higher than that of the traditional numerical method. It also has the advantage of simultaneously solving the boundary and control domain values.

(2) The multivariate quadratic radial basis function point collocation method is based on the principle of interpolation. The solution process does not require numerical integration calculation, the numerical simulation is easier to implement, and the result of calculating the free surface of seepage in unsteady conditions is more reasonable.

(3) This study solves the seepage free surface problem. The result value of the overflow point of the saturation line is anastomosis with the experimental value. The calculation is simple and has certain practical value in engineering calculations.

\section{Acknowledgments}

This paper is derived from the Fujian Water Conservancy Science and Technology Project.

\section{References}

1. CHAI, J.R. (2003) Nonlinear problems of rock and soil hydraulics. Rock and Soil mechanics, (S2): 159-162.

2. Mai-Duy N, Dalal D, et al. (2008)A symmetric integrated radial basis function method for solving differential equations. Numerical Methods for Partial Differential Equations, 34: 959-981.

3. MAO, C.X. (1990) Seepage calculation analysis and control. Water Conservancy and electric power press, Beijing

4. XUE Xin, JIN Wu-gen, LI Jue, et al. (2005)Trefftz direct method for solving free surface problems of seepage flow. Chinese Journal of Rock Mechanics and Engineering, 24 (13): 002322-2326. 